\title{
Universal twinkling exponents for spectral fluctuations associated with mixed chaology
}

\author{
By M. V. BerRy ${ }^{1}$, J. P. Keating ${ }^{2}$ And H. SChomerus ${ }^{3}$ \\ ${ }^{1}$ H. H. Wills Physics Laboratory, University of Bristol, \\ Tyndall Avenue, Bristol BS8 1TL, UK \\ ${ }^{2}$ School of Mathematics, University of Bristol, University Walk, \\ Bristol BS8 1TW, UK and Basic Research Institute in the Mathematical Sciences, \\ Hewlett-Packard Laboratories Bristol, Filton Road, \\ Stoke Gifford, Bristol BS34 8QZ, UK \\ ${ }^{3}$ Instituut-Lorentz, Universiteit Leiden, 2300RA Leiden, \\ The Netherlands
}

Received 2 February 2000; accepted 27 April 2000

For systems that are neither fully integrable nor fully chaotic, bifurcations of periodic orbits give rise to semiclassically emergent singularities in the fluctuating part $\mathcal{N}_{\mathrm{fl}}$ of the energy-level counting function. The bifurcations dominate the spectral moments

$$
M_{m}(\hbar)=\left\langle\left(\mathcal{N}_{\mathrm{fl}}\right)^{2 m}\right\rangle
$$

in the limit $\hbar \rightarrow 0$. We argue that $M_{m}(\hbar) \sim$ const. $/ \hbar^{\nu_{m}}$, and calculate the twinkling exponents $\nu_{m}$ as the result of a competition between bifurcations with different codimensions and repetition numbers.

Keywords: spectral statistics; quantum chaos; bifurcations; eigenvalues; asymptotics; fluctuations

\section{Introduction}

Our aim here is to characterize energy-level fluctuations in quantum systems whose classical counterparts are mixed, that is, neither completely integrable nor completely chaotic. Previous work (Berry \& Robnik 1984) indicates that the short-range fluctuations can be usefully approximated by a superposition of the Poisson and random-matrix spectral statistics that, respectively, describe integrable and chaotic systems (Berry 1983, 1987; Bohigas et al. 1984; Bohigas \& Giannoni 1984). Here we will argue that there is a complementary description, more fundamentally associated with the mixed regime.

The new description is associated with bifurcations, where combinations of stable and unstable orbits collide and transform into others, or annihilate, as a parameter (for example, energy) varies - it is the ubiquity of bifurcations, after all, that characterizes mixed systems. The main result will be the prediction that the spectral moments - describing the fluctuations in the distribution of energy levels as explained below - are dominated by a competition among the different sorts of bifurcation. 
Bifurcations are singularities of the dynamics, and the statistics to be calculated here are a new example of the wider class of 'singularity-dominated strong fluctuations'. This old but still-unfamiliar idea is that some variables exhibit wild (nonGaussian) fluctuations, with very large values described by scaling laws and associated with particular geometric singularities. For a review, see Berry (2000) (but note that some of the exponents in $\S 4$ of this publication are wrong, and are superseded by the present paper). The fluctuations most closely analogous to those we consider here are the intensity variations of twinkling starlight, where the short-wave singularities are caustics, and the intensity moments depend on a competition (Berry 1977) among catastrophes (universality classes of caustic). Although the formal analogy between spectral fluctuations and light caustics is close, orbit bifurcations are classified differently, and in their technical aspects, and their results, the two theories diverge.

Like all statistics related to chaology, those described here emerge semiclassically, that is, in the limit of vanishing Planck's constant $\hbar$. Consider a set of levels $\left\{E_{1}(\hbar), E_{2}(\hbar), \ldots, E_{j}(\hbar), \ldots\right\}$. This spectrum can be characterized by the counting function, or spectral staircase

$$
\mathcal{N}(E, \hbar)=\sum_{j=1}^{\infty} \Theta\left(E-E_{j}(\hbar)\right),
$$

where $\Theta$ denotes the unit step. As usual, we separate $N$ into its smooth and fluctuating parts,

$$
\mathcal{N}(E, \hbar)=\mathcal{N}_{\mathrm{sm}}(E, \hbar)+\mathcal{N}_{\mathrm{fl}}(E, \hbar) .
$$

$\mathcal{N}_{\mathrm{sm}}$ is given by the Weyl rule plus $\hbar$-corrections (Baltes \& Hilf 1976).

We will concentrate on the semiclassical size of the spectral fluctuations $\mathcal{N}_{\mathrm{fl}}$, as embodied in the spectral moments

$$
M_{m}(\hbar)=\left\langle\left[\mathcal{N}_{\mathrm{fl}}(E, \hbar)\right]^{2 m}\right\rangle .
$$

Here, $\langle\cdot\rangle$ denotes a local energy average for an individual Hamiltonian (assumed non-scaling). However, central to our calculation will be replacement of the energy average by averages over parameters for families of Hamiltonians including the given one. (For scaling systems, e.g. billiards, averaging over at least one parameter that changes the topology of the orbits is essential.) This implied ergodicity is implicit in many semiclassical arguments (for example, it leads directly to the short-range level repulsion for different classes of system (Berry 1983)). The main result will be that

$$
M_{m}(\hbar) \sim \frac{\text { const. }}{\hbar^{\nu_{m}}} \quad(\text { up to logarithms) as } \hbar \rightarrow 0,
$$

where $\nu_{m}$ are the 'twinkling exponents': universal numbers that we will determine by studying the hierarchy of bifurcations. Each exponent can be determined as the slope on a log-log plot, that is,

$$
\lim _{\hbar \rightarrow 0} \frac{\partial \log \left\{M_{m}(\hbar)\right\}}{\partial \log \{1 / \hbar\}}=\nu_{m} .
$$

Our calculations will be for systems with two freedoms. For these, a strict upper bound $\nu_{m} \leqslant 4 m$ follows from the Weyl rule

$$
\mathcal{N} \sim \mathcal{N}_{\text {sm }} \sim \text { const. } / \hbar^{2},
$$


implying

$$
\hbar^{2} \mathcal{N}_{\mathrm{fl}} \rightarrow 0 \quad \text { as } \hbar \rightarrow 0
$$

\section{Semiclassical theory}

To see the importance of bifurcations, we first recall the trace formulae, in which $\mathcal{N}_{\mathrm{fl}}(E)$ can be represented semiclassically as a sum over periodic orbits. In the generic case, where the orbits are isolated, the sum is over primitive periodic orbits $p$ with energy E, and their repetitions $r$ (Gutzwiller 1971; Balian \& Bloch 1972),

$$
\mathcal{N}_{\mathrm{fl}}(E, \hbar) \approx \frac{1}{\pi} \sum_{p} \sum_{r=1}^{\infty} \frac{\sin \left\{S_{p, r}(E) / \hbar-\mu_{p, r}\right\}}{r \sqrt{\left|\operatorname{det}\left(\left[\boldsymbol{M}_{p}(E)\right]^{r}-1\right)\right|}} .
$$

Here, $S_{p, r}$ is the action of the orbit, $\boldsymbol{M}_{p}$ is the monodromy matrix describing the linearized return map on the Poincaré section and $\mu_{p, r}$ is proportional to the Maslov index (which will play no further part our reasoning).

In the integrable case, for two freedoms, with Hamiltonian $H(\boldsymbol{I})$ involving action variables $\boldsymbol{I}=\left\{I_{1}, I_{2}\right\}$, the sum is over resonant tori characterized by their winding numbers $\boldsymbol{W}=\left\{W_{1}, W_{2}\right\}$, and the trace formula is (Berry \& Tabor 1976)

$$
\mathcal{N}_{\mathrm{fl}}(E, \hbar) \approx \frac{1}{\pi \hbar^{1 / 2}} \sum_{\boldsymbol{W}} \frac{\sin \left\{2 \pi \boldsymbol{W} \cdot \boldsymbol{I}_{\boldsymbol{W}}(E) / \hbar-\mu_{\boldsymbol{W}}\right\}}{|\boldsymbol{W}|^{3 / 2} \sqrt{K\left(\boldsymbol{I}_{\boldsymbol{W}}(E)\right)}} .
$$

Here, $\boldsymbol{I}_{\mathbf{W}}(E)$ are the actions of resonant tori, where the frequencies $\boldsymbol{\omega}$ are commensurate, and $K$ is the curvature of the energy contour $H(\boldsymbol{I})=E$ in $\boldsymbol{I}$ space.

When these formulae apply, there are no strong fluctuations and the estimation of $\mathcal{N}_{\mathrm{fl}}$ is fairly simple. For the chaotic case $(2.1)$, the prefactor is of order $\hbar^{0}$; the sum diverges, but can be regularized by truncation at orbits with period equal to the Heisenberg time $\hbar /$ (mean level spacing), which, together with the exponential proliferation of orbits with increasing period, leads to

$$
\left|\mathcal{N}_{\mathrm{fl}}\right| \sim \text { const. } \times \sqrt{\log (1 / \hbar)},
$$

and moments (1.5) with all twinkling exponents $\nu_{m}=0$. For the two-dimensional integrable case (2.2), where the sum converges,

$$
\left|\mathcal{N}_{\mathrm{fl}}\right| \sim \text { const. } \times \hbar^{-1 / 2}
$$

and (1.3) and (1.5) give $\nu_{m}=m$.

Here we are interested in cases when the trace formulae fail. This happens at bifurcations of periodic orbits. In (2.1), bifurcations of isolated orbits correspond to a unit eigenvalue of the monodromy matrix, so $\operatorname{det}(\boldsymbol{M}-1)$ vanishes, and the terms representing those orbits diverge. In (2.2), bifurcations of tori correspond to coalescence of parallel normals to the energy surface, so that $K$ vanishes and the terms representing those orbits diverge. The formulae fail, but it is clear that bifurcations lead to large values of $\mathcal{N}_{\mathrm{ff}}$. How large? This has been studied by several authors (Ozorio de Almeida \& Hannay 1987; Tomsovic et al. 1995; Ullmo et al. 1996; Sieber 1996; Schomerus \& Sieber 1997; Schomerus 1998; Sieber \& Schomerus 1998), who have found corrected versions of the trace formula that incorporate the bifurcations 
properly, with the result that $\mathcal{N}_{\mathrm{fl}}$ does not diverge but rises to values that increase as $\hbar \rightarrow 0$. We will extend these results to estimate the moments $M_{m}(\hbar)$ and hence the twinkling exponents $\nu_{m}$.

Near bifurcations, the trace formulae (2.1) and (2.2) must be replaced by the 'diffraction integrals' for which they are the stationary-phase approximations. Before writing these, we note that, for two freedoms, periodic orbits are fixed points of the map determined by successive intersections of the Poincaré section with coordinates $q, p$. In terms of the generating function $\phi$, the map can be specified as

$$
\left(\begin{array}{l}
q \\
p
\end{array}\right) \rightarrow\left(\begin{array}{l}
q^{\prime} \\
p^{\prime}
\end{array}\right) \quad \text { with } q=\partial_{p} \phi\left(q^{\prime}, p\right), \quad p^{\prime}=\partial_{q^{\prime}} \phi\left(q^{\prime}, p\right) .
$$

Thus periodic orbits are critical points of the reduced generating function (henceforth called the generator)

$$
\left.\begin{array}{c}
\Phi\left(q^{\prime}, p\right)=\phi\left(q^{\prime}, p\right)-q^{\prime} p, \\
\left\{\partial_{q} \Phi(q, p)=\partial_{p} \Phi(q, p)=0\right\} \leftrightarrow\left\{q^{\prime}=q, p^{\prime}=p\right\} .
\end{array}\right\}
$$

To write the diffraction integral describing semiclassical spectral fluctuations, we need the generator $\Phi_{r}$ for the $r$-times-iterated Poincaré map. $\left(\Phi_{r}(q, p ; E)\right.$ can be regarded as an effective Hamiltonian describing the motion between $r$ intersections of orbits with the Poincaré section.)

Up to irrelevant factors, the fluctuations are (Ozorio de Almeida \& Hannay 1987; Sieber 1996)

$$
\begin{aligned}
\mathcal{N}_{\mathrm{fl}}(E) & \approx \sum_{r=1}^{\infty} \mathcal{N}_{\mathrm{fl}, r}(E), \\
\mathcal{N}_{\mathrm{fl}, r}(E) & \left.=\operatorname{Im} \frac{1}{\hbar} \iint \mathrm{d} q \mathrm{~d} p \exp \left\{\frac{\mathrm{i}}{\hbar} \Phi_{r}(q, p ; E)\right\} \cdot\right\}
\end{aligned}
$$

Provided the sum over $r$ converges, this semiclassical theory implies the bound $\nu_{m}=2 m$, sharper than the strict bound $\nu_{m}=4 m$ obtained at the end of $\S 1$.

Periodic orbits correspond to stationary values of the phases in these integrals. If the stationary points are isolated, the stationary-phase approximation reproduces (2.1). If the system is integrable, $\Phi$ depends not on $q$ and $p$ separately, but on a combination (action variable) such as $q^{2}+p^{2}$, and is stationary on lines, corresponding to resonant tori; then stationary phase reproduces (2.2). At bifurcations, where isolated periodic orbits or resonant tori coalesce, these approximations fail. For different sorts of bifurcation, the patterns of coalescence are different, and $\mathcal{N}_{\mathrm{fl}}$ can be described locally (Ozorio de Almeida \& Hannay 1987) by replacing $\Phi$ by an appropriate normal form (the validity of the description can be extended by approximating the integrals (2.7) by the technique of uniform approximation (Sieber 1996; Schomerus \& Sieber 1997; Sieber \& Schomerus 1998), but that is not required for our purposes).

\section{Normal forms and scaling}

We envisage that for each bifurcation the local generator for each repetition number $r$ depends on parameters $\boldsymbol{x}=\left\{x_{n}\right\}(1 \leqslant n \leqslant K)$, in addition to $q$ and $p$; one 
of these parameters is the energy $E$, and $K$ is the codimension of the singularity. The parameters describe the unfolding of the bifurcation, that is, the ways in which the degenerate periodic orbit can split into combinations of non-degenerate orbits. Reflecting this, we denote the normal forms for the bifurcations by

$$
\Phi_{r}=\Phi_{r, K}(q, p ; \boldsymbol{x}),
$$

and define the associated canonical integrals

$$
\mathcal{N}_{\mathrm{fl}, r, K}(\boldsymbol{x}, \hbar) \equiv \operatorname{Im} \frac{1}{\hbar} \iint \mathrm{d} q \mathrm{~d} p \exp \left\{\frac{\mathrm{i}}{\hbar} \Phi_{r, K}(q, p ; \boldsymbol{x})\right\} .
$$

The strategy now is to simplify the $\hbar$ dependence of these integrals in a way that enables the averages in the moments (1.3) to be estimated as integrals over the parameters $\boldsymbol{x}$. This will be achieved by a two-stage process: rescaling the integration variables $q$ and $p$ to remove the $1 / \hbar$ factor from the dominant term (germ) of the generator in the exponent of (3.2), and then applying compensating rescaling of the parameters $\boldsymbol{x}$. This will lead to

$$
\mathcal{N}_{\mathrm{fl}, r, K}(\boldsymbol{x}, \hbar)=\frac{1}{\hbar^{\beta_{r, K}}} \mathcal{N}_{\mathrm{fl}, r, K}\left(\left\{x_{n} / \hbar^{\sigma_{n, r, K}}\right\}, 1\right) .
$$

The exponent $\beta$ describes the semiclassical strength of the spectral fluctuations at the bifurcation. The exponents $\sigma$ describe the scale of the interference fringes associated with non-degenerate periodic orbits that appear in the different unfolding directions $x_{n}$. We will also need the associated exponent

$$
\gamma_{r, K}=\sum_{n=1}^{K} \sigma_{n, r, K}
$$

describing the scaling of the $K$-dimensional $\boldsymbol{x}$ space hypervolume associated with interference near the bifurcation.

Armed with the scaling law (3.3), we can estimate the contribution of the bifurcation $r, K$ to the ensemble average for the $m$ th moment (1.3). This is

$$
\begin{aligned}
M_{m, r, K}(\hbar) & \equiv B \int \mathrm{d}^{K} \boldsymbol{x} \mathcal{N}_{\mathrm{fl}, r, K}(\boldsymbol{x}, \hbar)^{2 m} \\
& =\frac{B}{\hbar^{\left(2 m \beta_{r, K}-\gamma_{r, K}\right)}} \int \mathrm{d}^{K} \boldsymbol{y}\left[\mathcal{N}_{\mathrm{fl}, r, K}(\boldsymbol{y}, 1)\right]^{2 m},
\end{aligned}
$$

where $B$ is a normalization constant.

With the $\hbar$ dependence thus extracted, these contributions can now be compared for the different bifurcations. The dominant contribution(s) will come from the bifurcation(s) with the strongest $\hbar$ dependence, leading to the fluctuation moment scaling law (1.4) and (1.5) as the result of a competition among bifurcations, resulting in the twinkling exponents

$$
\nu_{m}=\max _{(r, K)}\left(2 m \beta_{r, K}-\gamma_{r, K}\right) .
$$

Here we will consider only the fully generic situation where the dynamics is such that all bifurcations occur in the neighbourhood of the system under consideration. 
Then the competition in (3.6) is unrestricted. If, for some reason (e.g. hyperbolicity), some classes of generic bifurcation are forbidden, or, because of symmetry, some nongeneric classes are allowed, the competition must be appropriately modified and the resulting exponents will be different. Analogous restricted competitions have been explored in the optical context (Walker et al. 1983) in the analysis of an experiment to measure twinkling exponents.

To carry out this programme, we need the normal forms for the bifurcations labelled $r, K$. For $r=1$, these are the elementary catastrophe polynomials representing the different ways that critical points of smooth functions can coalesce (Poston \& Stewart 1978; Arnold 1973, 1974, 1975), and the exponents in the scaling law (3.3) and (3.4) have already been calculated (Berry 1977). This is analogous to the optical case, where the appropriate diffraction integral is the first term $r=1$ of the sum (2.7). For the cuspoid catastrophes, where one variable ( $p$, say) is quadratic - in the language of singularity theory, these are catastrophes of corank 1-the normal forms are

$$
\Phi_{1, K}(q, p ; \boldsymbol{x})=p^{2}+q^{K+2}+\sum_{n=1}^{K} x_{n} q^{n}
$$

(any term of order $q^{K+1}$ can be eliminated by shifting the origin) and the exponents are

$$
\beta_{1, K}^{\text {cuspoids }}=\frac{K}{2(K+2)}, \quad \sigma_{n, 1, K}^{\text {cuspoids }}=1-\frac{n}{K+2}, \quad \gamma_{1, K}^{\text {cuspoids }}=\frac{K(K+3)}{2(K+2)} .
$$

We do not give the more complicated expressions corresponding to catastrophes of corank 2, where the generators involve both $q$ and $p$ non-trivially.

When $r>1$, however, the normal forms are not the elementary catastrophes, because the period- $r$ generator must have the special property of possessing an $r$ th root, namely the generator for the primitive map. Some information is available for bifurcations of period- $r$ orbits with $K=1$ (Meyer 1970, 1986; Arnold 1978; Ozorio de Almeida 1988) and $K=2$ (Schomerus 1998), but this is not sufficient for our purposes.

To get the results we need, we start by transforming to polar coordinates in phase space, that is,

$$
q=\sqrt{\frac{1}{2} I} \cos \phi, \quad p=\sqrt{\frac{1}{2} I} \sin \phi,
$$

and noting that the generators for period- $r$ bifurcations must have $\phi$ dependence with period $2 \pi / r$, so the $\phi$-dependent terms of lowest degree in $I$ must involve $\cos (r \phi)$ and $\sin (r \phi)$. Moreover, the generators must be smooth functions of $q$ and $p$ at the origin, which excludes terms $I^{s} \cos (r \phi)$ with $s<\frac{1}{2} r$. This leads to the surprising conclusion that if $r \geqslant 2 K+2$, the $\phi$-dependent terms are all of higher order than the unfolding terms containing the parameters $\boldsymbol{x}$. Thus we can write

$$
\Phi_{r, K}(q, p ; \boldsymbol{x})=I^{K+1}+\sum_{n=1}^{K} x_{n} I^{n}, \quad r \geqslant 2 K+2 .
$$

(The resemblance to the cuspoid generators (3.7) is misleading: it is not legitimate to eliminate the highest unfolding term $x_{K} I^{K}$ by shifting the origin of $I$, since this 
Table 1. Twinkling exponents $\nu_{m}$, and the codimension(s) $K$ of the dominating bifurcation(s), for generic two-freedom systems

$\begin{array}{lccccccccccc}m & 2 & 3 & 4 & 5 & 6 & 7 & 8 & 9 & 10 & 11 & 12 \\ \nu_{m} & \frac{5}{3} & 3 & \frac{9}{2} & 6 & \frac{38}{5} & \frac{46}{5} & \frac{65}{6} & \frac{25}{2} & \frac{85}{6} & \frac{111}{7} & \frac{123}{6} \\ \text { dominating } K & 2 & 2,3 & 3 & 3,4 & 4 & 4 & 5 & 5 & 5 & 6 & 6\end{array}$

would violate the condition that $I$ must be non-negative - alternatively stated, the origin of $I$ is privileged, unlike the origin of $q$ in (3.7).)

Reverting to $q$ and $p$, and scaling the integrals (3.2), we get, for the exponents in (3.3) and (3.4),

$$
\beta_{r, K}=\frac{K}{K+1}, \quad \sigma_{n, r, K}=1-\frac{n}{K+1}, \quad \gamma_{r, K}=\frac{1}{2} K, \quad r \geqslant 2 K+2 .
$$

Note that these exponents do not involve $r$.

In (3.10), we are neglecting the $\phi$-dependent terms, but we are not asserting their absence - of course, these terms must be present, to describe the 'island necklaces' of stable and unstable orbits into which the degenerate orbits bifurcate. But because the neglected terms are of higher order (reflecting the fact that the islands are very thin close to the bifurcation), the parameters that would multiply them acquire negative exponents $\sigma$ under scaling, and so disappear semiclassically from the diffraction integrals. Alternatively stated in the language of critical phenomena, these parameters are irrelevant variables. As a simple illustrative example, consider $K=1, r=5$ (i.e. $r>2 K+2$ ). Then the generator, including the leading $\phi$-dependent term, is

$$
\begin{aligned}
\Phi_{5,1}(q, p ; \boldsymbol{x}) & =I^{2}+x_{1} I+x_{5} I^{5 / 2} \cos (5 \phi) \\
& =4\left(p^{2}+q^{2}\right)^{2}+2 x_{1}\left(p^{2}+q^{2}\right)+2^{5 / 2} x_{5} \operatorname{Re}(q+\mathrm{i} p)^{5} .
\end{aligned}
$$

Scaling $\hbar$ from the exponent in (3.2) gives $\beta=\frac{1}{2}$, and incorporating this into the parameter $x_{1}$ gives $\sigma_{1}=\frac{1}{2}$. However, applying the same scaling to the 'necklace' parameter $x_{5}$ gives $\sigma_{5}=-\frac{1}{4}$, which is negative and therefore irrelevant. (If $r=$ $2 K+2$, the leading necklace parameter gives the marginal exponent $\sigma=0$, which does not affect any of our subsequent arguments.)

We have not determined the generators for $1<r<2 K+2$, but will soon argue that these bifurcations cannot contribute to the twinkling exponents.

\section{Battle of the bifurcations}

Suppose, for the moment, that all relevant bifurcations have $r \geqslant 2 K+2$, so that (3.10) applies. Then the twinkling exponents are determined by the competition (3.6), where the entrants are the $\beta$ and $\gamma$ values in (3.11), that is,

$$
\nu_{m}=\max _{K}\left(\frac{2 m K}{K+1}-\frac{1}{2} K\right) .
$$

The results are given in table 1.

Now we will argue that these results are unaffected by allowing the bifurcations with $r<2 K+2$ to enter the competition. This requires the twinkling exponents associated with this class of singularities to be smaller than those in table 1 . The 
generators for $r<2 K+2$ will contain $\phi$-dependent unfolding terms, and can be written in the form

$$
\Phi_{r, K}(q, p ; \boldsymbol{x})=I^{l(K)+1}+\sum_{n=1}^{l(K)} x_{n} I^{n}+\sum_{n=l(K)+1}^{K}(\text { terms involving } \phi),
$$

where $l(K)<K$ and all the terms involving $\phi$ are of lower degree than $I^{l(K)+1}$. For each such generator, there is a partner in the class with $r \geqslant 2 K+2$, of the form (3.10), with codimension $K^{\prime}=l(K)$. This partner has the same germ $I^{l(K)+1}$ as (4.2), and therefore the same exponent $\beta$, but its $\gamma$ exponent is smaller, because of the additional terms in (4.2). Therefore, the partner with $r \geqslant 2 K+2$ has the larger twinkling exponent $2 m \beta-\gamma$, and so dominates in the competition.

This general argument can be verified directly for the special case of the bifurcations with $r=1$, namely the elementary catastrophes. For the cuspoids (corank 1), with exponents (3.8), it is easy to calculate the results of the competition (3.6); this has already been done in the optical context (Berry 1977), and all the exponents are indeed smaller than those in table 1 . The same is true for the corank 2 catastrophes, even though the exponents are all larger than for corank 1 (for corank 2, the classification is incomplete, but the conclusion holds for all classes of singularity that have been examined).

Thus the entries in table 1 are confirmed as the universal twinkling exponents associated with bifurcations of generic systems with two freedoms.

\section{Discussion}

We have argued that to leading order in $1 / \hbar$, the spectral fluctuation moments $M_{m}$ diverge according to power-laws, with exponents - those winning the competition (3.6) - given in table 1. This goes far beyond the already established fact that bifurcations contribute to the spectral statistics when regular and chaotic orbits coexist (Berry et al. 1998), because these new semiclassical fluctuation phenomena involve many competing bifurcations, not just one.

Two observations may assist the eventual observation of the universal fluctuations we are predicting. The first relates to our concentration on the average effect of large spectral fluctuations associated with individual bifurcations of periodic orbits of finite length, while ignoring possible collective effects of long orbits. But this collective effect seems small (equation (2.3) and the remark following it), so we expect the associated fluctuations to contribute only a weak background that will not mask the bifurcation fluctuations we are interested in. However, we were not able to make a definitive assessment of possible collective effects of bifurcations amongst the long orbits.

The second observation is that, although all the twinkling exponents in table 1 satisfy the inequality $\nu_{m}<2 m$, all the exponents with $m>3$ exceed the value $\nu_{m}=m$ for integrable systems, rendering unnecessary the problematic subtraction of possible contributions to the fluctuations from the (non-resonant) KAM tori that persist in the systems we have been studying here.

Nevertheless, it is difficult to make quantitative predictions of the circumstances in which the twinkling exponents might be seen in computer or laboratory experiments. To illustrate this, suppose that the dominant bifurcation, with exponent $\nu_{m}$, 
has an associated coefficient $A_{m}$, and the runner-up in the competition has exponent $\nu_{1 m}<\nu_{m}$ and coefficient $A_{1 m}$. Then the two leading terms of the moment asymptotics will be

$$
M_{m}(\hbar) \sim \frac{A_{m}}{\hbar^{\nu_{m}}}+\frac{A_{1 m}}{\hbar^{\nu_{1 m}}} .
$$

If it should happen that $A_{m} \ll A_{1 m}$, and $\nu_{m}$ exceeds $\nu_{1 m}$ only slightly, experiments will indicate the wrong exponents $\nu_{1 m}$ unless $\hbar$ is less than the crossover value

$$
\hbar=\left(A_{m} / A_{1 m}\right)^{1 /\left(\nu_{m}-\nu_{1 m}\right)},
$$

which in the circumstances indicated is very small.

Our reasoning hints at fabulous complexity in the full semiclassical asymptotics of the moments: many different bifurcations contribute according to (3.5), and these terms are merely the leading orders in (almost certainly divergent) $\hbar$ expansions, because the normal forms of the generators give only local approximations to the diffraction integrals (2.7), which themselves are lowest-order semiclassical approximations. These observations lead to the expectation that

$$
M_{m}(\hbar)=\sum_{r, K} \frac{A_{m, r, K}}{\hbar^{\left(2 m \beta_{r, K}-\gamma_{r, K}\right)}}\left(1+\sum_{s=0}^{\infty} \alpha_{s, m, r, K} \hbar^{s}\right)
$$

(where the $A$ and $\alpha$ coefficients might involve logarithms of $1 / \hbar$ ).

We know nothing about the leading-order bifurcation coefficients $A_{m, r, K}$ or the corrections $\alpha_{s, m, r, K}$. These coefficients are not universal, so calculating them would require detailed knowledge of the individual bifurcations in the particular dynamical system being considered. Even for the less complicated case of optical twinkling, the $A$ coefficients have been calculated only for the simplest situation: cuspoids in diffraction from a corrugated phase-changing screen (Hannay 1982, 1983).

H.S. was supported by NWO/FOM (The Netherlands).

\section{References}

Arnold, V. I. 1973 Normal forms for functions near degenerate critical points, the Weyl groups of $A_{k}, D_{k}, E_{k}$ and Lagrangian singularities. Funct. Analysis Appl. 6, 254-272.

Arnold, V. I. 1974 Normal forms of functions in neighbourhoods of degenerate critical points. Russ. Math. Surv. 29, 10-50.

Arnold, V. I. 1975 Critical points of smooth functions and their normal forms. Russ. Math. Surv. $30,1-75$.

Arnold, V. I. 1978 Mathematical methods of classical mechanics. Springer.

Balian, R. \& Bloch, C. 1972 Distribution of eigenfrequencies for the wave equation in a finite domain. III. Eigenfrequency density oscillations. Ann. Phys. 69, 76-160.

Baltes, H. P. \& Hilf, E. R. 1976 Spectra of finite systems. Mannheim: Bibliographisches Institut.

Berry, M. V. 1977 Focusing and twinkling: critical exponents from catastrophes in non-Gaussian random short waves. J. Phys. A 10, 2061-2081.

Berry, M. V. 1983 Semiclassical mechanics of regular and irregular motion. In Les Houches Lecture Series (ed. G. Iooss, R. H. G. Helleman \& R. Stora), vol. 36, pp. 171-271 Amsterdam: North-Holland.

Berry, M. V. 1987 The Bakerian Lecture: Quantum chaology. Proc. R. Soc. Lond. A 413, 183198. 
Berry, M. V. 2000 Spectral twinkling. In Enrico Fermi school. New directions in quantum chaos (ed. G. Casati, I. Guarneri \& U. Smilansky). Varenna: Italian Physical Society. (In the press.)

Berry, M. V. \& Robnik, M. 1984 Semiclassical level spacings when regular and chaotic orbits coexist. J. Phys. A 17, 2413-2421.

Berry, M. V. \& Tabor, M. 1976 Closed orbits and the regular bound spectrum. Proc. R. Soc. Lond. A 349, 101-123.

Berry, M. V., Keating, J. P. \& Prado, S. D. 1998 Orbit bifurcations and spectral statistics. J. Phys. A, 31, 245-254.

Bohigas, O. \& Giannoni, M. J. 1984 Chaotic motion and random-matrix theories. In Mathematical and computational methods in nuclear physics (ed. J. S. Dehesa, J. M. G. Gomez \& A. Polls), vol. 209, pp. 1-99. Springer.

Bohigas, O., Giannoni, M. J. \& Schmit, C. 1984 Characterization of chaotic quantum spectra and universality of level fluctuation laws. Phys. Rev. Lett. 52, 1-4.

Gutzwiller, M. C. 1971 Periodic orbits and classical quantization conditions. J. Math. Phys. 12, 343-358.

Hannay, J. H. 1982 Intensity fluctuations beyond a one-dimensional random refracting screen in the short-wave limit. Optica Acta 29, 1631-1649.

Hannay, J. H. 1983 Intensity fluctuations from a one-dimensional random wavefront. J. Phys. A 16, 61-66.

Meyer, K. R. 1970 Generic bifurcation of periodic points. Trans. Am. Math. Soc. 149, 95-107.

Meyer, K. R. 1986 Bibliographic notes on generic bifurcations in Hamiltonian systems. Contemp. Math. 56, 373-381.

Ozorio de Almeida, A. M. 1988 Hamiltonian systems: chaos and quantization. Cambridge University Press.

Ozorio de Almeida, A. M. \& Hannay, J. H. 1987 Resonant periodic orbits and the semiclassical energy spectrum. J. Phys. A 20, 5873-5883.

Poston, T. \& Stewart, I. 1978 Catastrophe theory and its applications. Boston, MA: Pitman.

Schomerus, H. 1998 Periodic orbits near bifurcations of codimension two: classical mechanics, semiclassics and Stokes transitions. J. Phys. A 31, 4167-4196.

Schomerus, H. \& Sieber, M. 1997 Bifurcations of periodic orbits and uniform approximations. J. Phys. A 30, 4537-4562.

Sieber, M. 1996 Uniform approximation for bifurcations of periodic orbits with high repetition numbers. J. Phys. A 29, 4715-4732.

Sieber, M. \& Schomerus, H. 1998 Uniform approximations for period-quadrupling bifurcations. J. Phys. A 31, 165-183.

Tomsovic, S., Grinberg, M. \& Ullmo, D. 1995 Semiclassical trace formulas of near-integrable systems: resonances. Phys. Rev. Lett. 75, 4346-4349.

Ullmo, D., Grinberg, M. \& Tomsovic, S. 1996 Near-integrable systems: resonances and semiclassical trace formulas. Phys. Rev. E 54, 136-152.

Walker, J. G., Berry, M. V. \& Upstill, C. 1983 Measurement of twinkling exponents of light focused by randomly rippling water. Optica Acta 30, 1001-1010. 